\title{
Rucaparib in patients presenting a metastatic breast cancer with Homologous Recombination Deficiency, without germline BRCA1/2 mutation
}

Anne Patsouris ( $\nabla$ anne.patsouris@ico.unicancer.fr)

institut de cancérologie de l'ouest-Pays de Loire https://orcid.org/0000-0001-9101-5424

M'boyba Khadija DIOP

Bioinformatic Plateform UMS AMMICA Gustave Roussy campus

Olivier Tredan

Centre Léon Bérard

Daniel Nenciu

Department of Radiology, Institut de Cancérologie de l'Ouest-Pays de La Loire

Anthony Goncalves

Departement of medical oncology, Institut Paoli-Calmettes

Monica Arnedos

departement of medical oncology, Gustave Roussy, Université Paris Saclay

Marie-Paule Sablin

departement of medical oncology, Curie institute

Pascal Jézéquel

Institut de Cancérologie de l'Ouest-Pays de La Loire

Marta Jimenez

Unicancer

Nathalie Droin

Gustave roussy https://orcid.org/0000-0002-6099-5324

Ivan Bieche

Institut Curie

Celine Callens

Department of Genetics, Curie Institute and Paris Sciences, Lettres University

Andrea Loehr

Translational Medicine Department, Clovis Oncology

Cecile Vicier

Institut Paoli-Calmettes

Valérie Seegers

Department of Biostatistics, Institut de Cancérologie de l'Ouest-Pays de La Loire

Thomas Filleron 
Department of Biostatistics, Claudius-Regaud Institute, IUCT-oncopole

\section{Fabrice André}

Institut Gustave Roussy https://orcid.org/0000-0001-5795-8357

\section{Article}

Keywords: breast cancer, rucaparib, HER2-negative metastatic breast cancer, germline BRCA1/2 mutation, PARP inhibitors

Posted Date: October 6th, 2020

DOI: https://doi.org/10.21203/rs.3.rs-78109/v1

License: (c) (1) This work is licensed under a Creative Commons Attribution 4.0 International License. Read Full License 


\section{Abstract}

Breast cancer may present genomic alterations leading to homologous recombination deficiency. PARP inhibitors have proved their efficacy in patients with HER2-negative metastatic breast cancer ( $\mathrm{mBC}$ ) harboring germline (g) BRCA1/2 mutations. We conducted the phase 2 RUBY trial to assess the efficacy of rucaparib in HER2-negative $\mathrm{mBC}$ with high genomic loss of heterozygosity ( $\mathrm{LOH})$ score or somatic, without $\mathrm{g} B R C A 1 / 2$ mutation. 220 of 711 patients with $\mathrm{mBC}$ screened for $\mathrm{LOH}$ presented high LOH score which was associated with a higher likelihood of death $(\mathrm{HR}=1.39,95 \% \mathrm{Cl}: 1.11-1.75, \mathrm{p}=0.005)$. The primary objective was not reached with a clinical benefit rate (objective response or SD>16 weeks) of $13.5 \%$. Two LOH-high patients, without somatic BRCA1/2 mutation, presented a complete and durable response (14 and 32 months). HRDetect tended to be associated with response to rucaparib, whithout reaching statistical significance (median HRDetect responders versus non responders: 0.465 versus, $0.040, p=0.2135$ ). Our data suggests that a small subset of patients with high LOH score could derive benefit from PARP inhibitors.

\section{Background}

Despite efforts in targeted therapeutic approaches over the past 20 years, metastatic breast cancer (mBC) remains a lethal disease with a median overall survival (OS) of 39.5 months and the need for further development in personalized medicine ${ }^{1}$. Breast cancer may present genetic alterations in the homologous recombination repair (HRR) pathway, a high fidelity repair mechanism for double stranded DNA breaks ${ }^{2}$. Breast cancer susceptibility genes 1 or $2(B R C A 1 / 2)$ are the most studied HRR genes. These alterations lead to an homologous recombination deficient (HRD) tumor and a dependency on the base excision repair (BER) system, a repair pathway for single stranded breaks, with a key role of the enzyme poly(ADPRibose) polymerase (PARP). This dependency may be exploited for therapeutic purposes: PARP inhibition in BRCA1/2 mutated cancer cells, causes unresolved DNA damage leading to cell death ${ }^{3}$. PARP inhibitors efficacy is also due to PARP trapping that results in replication arrest by blocking the replication fork 4 .

In the metastatic setting, the two PARP inhibitors olaparib and talazoparib have been approved in previously treated patients with HER2 negative (HER2-) mBC harboring a germline (g) BRCA1/2 mutation, following results of two open-label, multicenter, randomized, phase 3 trials testing PARP inhibitor versus treatment of physician's choice with improvement of progression-free survival (PFS) ${ }^{5-8}$. In these two trials, both triple-negative and estrogen receptor positive (ER+)/HER2- $\mathrm{mBC}$ patients benefited from PARP inhibitors. Finally, veliparib in combination with platinum-based chemotherapy showed recently its superiority compared to chemotherapy alone in terms of PFS (14.5 versus 12.6 months; $p=0.002)$ in patients with HER2 negative untreated $\mathrm{mBC}$ with $g B R C A 1 / 2$ mutation ${ }^{9}$.

However, germline or somatic (s) BRCA1/2 mutations occur in for less than $5 \%$ of breast cancer patients ${ }^{10,11}$. Furthermore, some breast cancers present HRD genotype without gBRCA1/2 mutation, (also called "BRCAness" profile) that share therapeutic vulnerabilities with these hereditary cancers, including 
PARP inhibitors sensitivity ${ }^{4,12}$. This encompasses somatic mutations in genes that modulate HRR pathway, resulting in a genomic instability, reflective of the preferential use of the non-conservative double stranded DNA breaks repair pathway such as non-homologous end joining and single-strand annealing ${ }^{12}$. Genomic instability scores like telomeric allelic imbalance score $(\mathrm{TAl})^{13}$, loss of heterozygoty $(\mathrm{LOH})^{14}$, and large-scale state transition (LST $)^{15}$ have been associated with several characteristics of a BRCAness profile. HRD profiling has also been approached by mutational signatures, a genomic scar that corresponds to a pattern of mutations, strongly associated with BRCA1/2 mutations ${ }^{16}$. Recently, NickZainal et a/ developed HRDetect, a whole genome sequencing predictor based on mutational signatures, to investigate HRD profiles among 560 breast carcinomas. They identified $22 \%$ of patients with a HRD profile that could benefit from PARP inhibitors ${ }^{17}$. A phase 2 trial has shown activity of talazoparib in somatic or germline HRR mutated genes in HER2- breast cancer patients. Three of 12 patients presented a tumor response (2 gPALB2, 1 gCHEK2/gFANCA/ sPTEN) and 3 additional patients ( $g P A L B 2, s A T R$, $S P T E M$ ) had stable disease $\geq 6$ months $^{18}$. Another phase 2 trial, the TBCRC 048 study, has recently shown activity of olaparib in patients that harbored either germline or somatic mutations in HRR pathway genes other than $B R C A 1 / 2$ or with $B R C A 1 / 2$ somatic mutations. In the germline cohort, only patients with gPALB2 mutations (9/27) presented a partial response under olaparib while the 8 out of 26 patients achieving a partial response in the somatic cohort harbored a $S B R C A 1 / 2$ mutation ${ }^{19}$.

Rucaparib, an oral, small-molecule inhibitor of PARP-1, PARP-2 and PARP-3, has demonstrated antitumor activity in various tumor types ${ }^{20,21}$. Rucaparib has been approved by the united States Food Drug and Administration (US FDA) and by the European Medicines Agency (EMA), for the treatment of patients with deleterious BRCA mutation-associated advanced ovarian cancer who have been treated with two or more therapies ${ }^{22}$ and recently by the US FDA for BRCA mutation associated metastatic castration-resistant prostate cancer ${ }^{21,23}$. Based on data from two open-label, single-arm, multicenter trials, ARIEL2 and Study 10, FDA also approved the companion diagnostic FoundationFocus CDx $\mathrm{BRCA}_{\mathrm{LOH}}$, that identifies ovarian HRD tumors by combining analysis of somatic BRCA1/2 mutation status and the percentage of genomewide $\mathrm{LOH}^{24-26}$.

The goal of the RUBY study was to evaluate the efficacy of rucaparib for the treatment of locally advanced or metastatic HER2- $\mathrm{mBC}$ with either high tumor genomic LOH score or somatic without germline $B R C A 1 / 2$ mutation. A second objective was to assess the prognostic value of high genomic $\mathrm{LOH}$ score.

\section{Results}

\section{PRONOSTIC VALUE OF HIGH GENOMIC LOH SCORE}

To identify patients for the RUBY study, we analyzed data from the SAFIR02-Breast and SAFIRTOR studies, as well as the publicly available TCGA breast cancer data set (TCGA BRCA). 711 patients $(n=620$ from SAFIR02-Breast, $n=91$ from SAFIRTOR studies) were successfully tested for a genomic LOH score. 
220 (30.9\%) presented a high genomic LOH score. We used SAFIR02-Breast data only to determine the prognostic value of the $\mathrm{LOH}$ score. Patients' characteristics are summarized in supplementary table 1 . All patients presented with $\mathrm{mBC}$, and genomic $\mathrm{LOH}$ score was determined on tumor material obtained when patients developed metastases. "High" genomic LOH was defined as having genomic $\mathrm{LOH}$ across $18 \%$ or more of the genome (cf. Materials and Methods). Among the 620 SAFIR02 LOH profiles, 206 (33.2\%) presented a high genomic $\mathrm{LOH}$ score. This proportion was lower in the overall population of the early stage TCGA BRCA dataset: 204/1004 (20,3\%). When analyzing by tumor subtypes, high LOH score comprised 107/359 (29.8\%) of the ER+/HER2- mBC from SAFIR02 compared to 63/456 (13.8\%) of early ER+/HER2- BC from TCGA BRCA. These findings suggest that ER+/HER2- $m B C$ present with higher HRD as compared to the early stage $\mathrm{BC}$. Interestingly, the opposite was found for the triple-negative (TN) population: $96 / 243$ (39.5\%) mBC as compared to 66/113 (58.4\%) early BC (table 1 ). In the metastatic setting, high genomic LOH score was associated with a higher rate of somatic (s) BRCA1/2 mutation compared to low LOH score ( $2.9 \%$ versus $0.5 \% \mathrm{p}=.0187$ ) (supplementary table 2 ). In a multivariate analysis, high LOH score was associated with worse overall survival in the overall SAFIR02 population (HR=1.39, 95\% confident interval [Cl] $\mathrm{Cl}: 1.11-1.75, \mathrm{p}=0.005)$ and in the subgroup of ER+/HER2- patients (adjusted $\mathrm{HR}=1.45 ; 95 \% \mathrm{Cl}, 1.06-1.99 ; \mathrm{p}=0.019$ ). In the TN mBC population, a similar trend for a worse prognosis in patients with high $\mathrm{LOH}$ score than patients with low $\mathrm{LOH}$ score, despite the lack of statistical significance (adjusted HR $=1.32 ; 95 \% \mathrm{Cl} ; 0.95-1.84 ; \mathrm{p}=0.095$ ) (Figure 1 and Supplementary table 3-5). In a univariate analysis, the site of metastatic biopsy (liver or not) did not affect the prognostic value of high LOH score.

\section{EFFICACY}

Between January 9, 2016 and December 15, 2018, 12 centers enrolled 42 patients into the RUBY study. Two patients were excluded before treatment, one for lack of measurable target lesion at inclusion (according to RECIST 1.1) and one for disease progression (brain metastasis) before treatment initiation, and 40 patients received at least one dose of rucaparib (Supplementary Figure 1).

The median age at inclusion was 54 years. Thirty-five patients (87.5\%) were included based on high genomic LOH score and 5 patients (12.5\%) on the basis of a sBRCA1/2 mutation. Details of the $s B R C A 1 / 2$ mutations are provided in supplementary table 6 . Tissue was obtained from liver biopsies in $18(51 \%)$ patients. Nineteen patients (47.5\%) had triple-negative breast cancer (TNBC), assessed on metastatic samples. Two patients, with an ER+/HER2- phenotype on their primary tumor, presented a TN phenotype on their metastatic samples. Thirty-four patients (84.6\%) presented visceral metastases including $29(74.4 \%)$ with liver metastasis. Ten patients received prior platinum salt treatment including 2 patients (5.0\%) in the adjuvant setting. Patients' characteristics are summarized in table 2.

The median duration of follow-up was 25.8 months ( $95 \% \mathrm{Cl}$ : 14.7-not reached). At the time of data cut-off (January 8, 2020), 37 patients (92.5\%) had stopped their treatment because of disease progression and 1 (2.5\%) due to toxicity. Two patients (5\%) were still on treatment, and 29 (72.5\%) patients had died. Of the 40 patients who received at least one dose of rucaparib, 4 patients left the trial before the first RECIST 
evaluation including 3 patients because of progression (considered as treatment failure in modified intent to treat population) and one patient due to toxicity.

At final analysis, five of the 37 evaluable patients showed a clinical benefit per investigator assessment, defined as described in the methods section, including one complete response, three partial responses and one stable disease $>16$ weeks. The centralized review confirmed 2 complete responses, 3 partial responses and 1 stable disease $>16$ weeks. Responders' characteristics are summarized in supplementary table 7. One patient showed a stable disease with a duration of 15.7 weeks, confirmed by central review, not included in the clinical benefit rate events ( $<16$ weeks). The per investigator clinical benefit rate was $13.5 \%$ (5/37 patients; $95 \% \mathrm{Cl}$ : 4.5-28.8). For some patients, rucaparib treatment produced an outlier tumor shrinkage. One patient harboring $S B R C A 1$ mutation showed a stable disease and one patient with a SBRCA2 mutation and a gPALB2 mutation showed a partial response (Figure 2). The median duration of rucaparib treatment was 47 days (range: 8-668 days). Median PFS was 1.7 months (95\% Cl: 1.4-1.8) and median OS was 6.7 months (95\% Cl: $5.6-12.5)$.

\section{SAFETY}

Of the 40 patients who received at least one dose rucaparib (safety population), 12 patients (30\%) had at least one dose reduction and $12(30 \%)$ a treatment interruption. Three patients discontinued treatment due to toxicity, including one patient who discontinued rucaparib consecutive to grade 3 anemia and thrombocytopenia before the first assessment of rucaparib efficacy. The other 2 patients who discontinued treatment due to toxicity presented a disease progression concomitant with grade 3 anemia and lymphopenia for the first patient, and a disease progression concomitant with grade 3 neutropenia and grade 4 thrombocytopenia for the second patient.

The most frequently reported adverse event (AE) ( $\geq 20 \%$ of the safety population) were asthenia $(71 \%)$, aspartate aminotransferase (AST) increase (66\%), gamma-glutamyltransferase (GGT) increase (58\%), anemia (48\%), alanine aminotransferase (ALT) increase (40\%), lymphopenia (38\%), blood alkaline phosphatase increase (34\%), nausea (36\%), thrombocytopenia (20\%) and hypercholesterolemia (20\%). Grade 3 AE were mostly related to liver enzyme disorders with AST (25\%), GGT (20\%), and ALT (15\%) increase. Grade 3 asthenia was reported by $18 \%$ of the patients. The most frequently reported grade $4 \mathrm{AE}$ were GGT increase (8\%), anemia and thrombocytopenia (5\%), and blood alkaline phosphatase increase and hypertriglyceridemia (3\%). Of note, 22 patients (55\%) had some degree of hepatic cytolysis at baseline. Twelve patients (30\%) had at least 1 serious $A E$, predominantly blood and lymphatic system disorders (5 patients) (supplementary table 8 ).

\section{HRDetect}

Of the 40 RUBY patients treated with rucaparib, 24 had tumor DNA available paired to constitutional DNA for whole genome analysis. The average depth for tumor sequencing was $23.19 \mathrm{X}$. This analysis included 2 patients that harbored a $s B R C A 1$ mutation, 1 patient with a $s B R C A 2$ mutation, 14 patients with 
ER+/HER2- phenotype and 10 patients with TN phenotype. The tumors samples of 5 of the 6 patients that presented a clinical benefit from rucaparib, according to centralized review, were assessed.

Among the five responders, two patients were identified as having a probability of BRCA $1 / 2$ deficiency per HRDetect exceeding 0.7: patient 1 (LOH score 19\%; HRDetect score: 0.72154 ) presented a complete response and is still under treatment (> 32months), and patient 7 ( $\mathrm{LOH}$ score $39 \%$; HRDetect score: $0.93522)$ presented a partial response. None of them were included on the basis of $s B R C A 1 / 2$ mutation. The other two responding patients harbored a probability of 0.465 (patient 4) and 0.024 ((patient 2), and presented a liver partial response (patient 4) and a stable disease, respectively. Finally, one patient with stable disease $>16$ weeks (Patient 3 ), included on the basis of $S B R C A 1$ mutation (c.171del (p.pro58leufs ${ }^{\star} 11$ ) harbored a low probability at $1.062 \mathrm{e}-4$. HRDetect score tended to be higher in patients that presented a benefit from rucaparib versus patients without clinical benefit rate, although not significant. The mean HRDetect score in responders versus non-responders was 0.465 versus $0.040(p=$ 0.2135). Probability of $B R C A 1 / 2$ deficiency exceeding 0.7 was significantly correlated with tumor response to rucaparib $(\mathrm{p}=0.0362)$ (Figure 3$)$. Patient 34 with $B R C A 2$ truncated mutation $(\mathrm{c} .1135 \mathrm{G}>\mathrm{T}, \mathrm{p}$. (Gly379Ter)) and patient 40 with BRCA1 truncated mutation (c.1762_1768del, p.(Ser588Valfs*2)) without any clinical response presented a low HRD probability of 0.002 and 0.012 , respectively).

\section{Discussion}

Results from the RUBY trial showed efficacy of rucaparib as monotherapy treatment in 6 patients either with $s B R C A 1 / 2$ mutation or high genomic $\mathrm{LOH}$, as assessed by centralized review, including an exceptional liver partial response and 2 complete responses with a duration of treatment of 14 and 32 months in patients enrolled on the basis of high LOH score. The RUBY trial did not meet its primary endpoint nevertheless our data support the potential benefit of a PARP inhibitor with prolonged responses in a subset of $\mathrm{mBC}$ patients beyond $g B R C A 1 / 2$ mutation.

Does the genomic scars captured by genomic scores, related to HRD or rather reflect of genome evolution and instability? The poor study outcomes could be explained by patient selection. Indeed, LOH score was used to select patients as a surrogate marker of HRD ${ }^{27}$. LOH scores have been developed in ovarian cancer and primary breast cancers ${ }^{25}$. Nevertheless, it is not clear whether this test applies to heavily pretreated $\mathrm{mBC}$. Indeed, as previously reported, genome evolve during drug resistance and metastatic process ${ }^{11,28}$. This genome evolution increases the percentage of genomic scars previously associated with HRD. Nevertheless, whether HRD creates these genomic scars, or if it is a marker of genome evolution and instability unrelated to HRD remains unclear. We previously showed that high genomic LOH was more frequent in ER+/HER2- mBC compared to TCGA ER+/HER2- breast cancer samples. The opposite was observed in TNBC, which could suggest that in this phenotype, tumor cells could not survive a high degree of genomic instability with selection of the tumor clones which escape adjuvant systemic treatment ${ }^{27}$. Furthermore, genomic LOH score does not allow for an accurate prediction of HRD genomic 
scar clonality, thus, therapeutic resistance can be associated with selective advantage of clonal selection during the course of the disease $e^{4}$.

\section{PARP inhibitors efficacy beyond gBRCA1/2 mutations}

Most of the current trials testing PARP inhibitors in non-germline BRCA1/2-mutated advanced breast cancer, like the phase 2 studies VIOLETTE (NCT03330847), NOBROLA (NCT03367689) and DOLAF (NCT04053322), are using a targeted next-generation sequencing panel of HRR genes considering the same contribution of all HRR genes to the HRD profile. Among patients without $g B R C A 1 / 2$ mutations, most of those benefiting from PARP inhibitors harbored $g P A L B 2$ mutations and, to a lesser extent, $s B R C A 1 / 2$ mutations. Indeed, the TBCRC 048 trial recently showed activity of olaparib in either gPALB2 mutation or in $S B R C A 1 / 2$ mutations but not with $A T M$ or $C H E K 2$ mutations ${ }^{29}$. In another phase 2, three of the 6 patients that benefited from talazoparib presented a gPALB2 mutation ${ }^{18}$. In RUBY, two of the five patients that harbored $\mathrm{s} B R C A 1 / 2$ mutation benefited from rucaparib with a stable disease (BRCA1 mutation) and a partial response (BRCA2 mutation). This last patient also presented a gPALB2 mutation. Of note, the two patients with prolonged complete response were selected on the basis of high genomic LOH score. However, genomic LOH analysis alone seems to be insufficient to completely predict HRD status $^{14}$. In the phase-3, ARIEL 3 trial, $30 \%$ of patients with BRCA wild-type and low-LOH ovarian carcinoma experienced clinical benefits for more than a year in the rucaparib group more than a year

versus $5 \%$ in the placebo group ${ }^{30}$. Similar benefits of rucaparib treatment were reported in ARIEL 2 trial $^{25}$.

\section{Clinical validity of HRDetect}

Exposure to different mechanisms of DNA damage and repair lead to specific patterns of mutations that constitute mutation signatures, with more than one process operative in most cancers ${ }^{16}$. The basesubstitution signature 3 has previously been shown to distinguish gBRCA1/2 deficient and more recently gPALB2 mutation (nonsense and frameshift missense) from sporadic cancer in a subset of breast cancer patients but is not discriminating enough when used alone ${ }^{31}$. Indeed, at least five signatures have been observed resulting from a $B R C A 1 / 2$ gene defect, including microhomology-mediated indels and more deletions especially those observed in BRCA1/2 mutant breast cancers, reflect of the preferential use of error-prone mechanisms of DSB repair such as non-homologous end-joining process ${ }^{32-34}$. The whole genome-based predictor, HRDetect, developed by Nick-Zainal et al, is based on a lasso logistic regression model applied on HRD copy number indices, twelve base-substitution, two indel and 6 mutational signatures previously extracted ${ }^{17}$. HRDetect was investigated on 560 breast carcinoma samples and was very performant to identify patients with HRD profile (up to $22 \%$ ) with a high sensitivity even in the case of low-coverage genome sequencing using an absolute probability cutoff of $0.7^{17}$. Most notably, HRDetect was more performant in identifying BRCA1/2 defective tumors than the combination of $\mathrm{LOH}$, LST and TAl forming the HRD-score (score $>42$ in HRD tumors) ${ }^{17,33}$. In the phase 2 window trial RIO $(n=43)$, HRDetect score $>0.7$ identified $69 \%(18 / 26)$ of untreated primary TNBC that harbored HR deficiency (HRDetect+) including identification of all tumors with genomic or epigenetic alteration in HRR 
pathways (somatic mutation of BRCA1, BRCA2 and PALB2, and promoter methylation of BRCA1 and $R A D 51 C)^{35}$. HRDetect+ tumors were associated with induction of RAD51 nuclear foci as assessed by immunochemistry and clinical activity of rucaparib with a greater circulating tumor DNA suppression after 15 days of treatment $(n=15, p=0.027)$. A phase 2 trial is currently evaluating olaparib associated with trastuzumab in HER2+ mBC that present either a gBRCA1/2 mutation or a HRDetect+ tumor (NCT039311551). In RUBY study, two patients with high genomic LOH were predicted to have BRCA1/2 deficiency with HRDetect $(>0.7)$, including one patient, that presents a complete response and still under treatment with a duration of response $>32$ months and one patient with a partial response. The patient with an important partial liver metastasis response presented a higher HRDetect score than most of nonresponders $(0.46)$. Only one patient with high genomic $\mathrm{LOH}$ and prolonged stable disease (>14 months) presented a low HRDetect score (0.02).

Overall, this study shows that a small subset of patients with HRD but without gBRCA1/2 mutation could benefit from PARP inhibition. Nevertheless, currently available testing are probably too broad, and there is a need to develop additional biomarkers to more selectively identify potential responders. HRDetect and specific mutations on HR pathways ( $g P A L B 2, s B R C A 1 / 2$ mutations) are candidate biomarkers. Further meta-analysis of current phase 2 trials will help better define which biomarker combination may accurately predict response to PARP inhibitors in patients without $g B R C A 1 / 2$ mutations.

\section{Material And Methods}

\section{Patients and Study Design}

RUBY was a single arm, open-label, multicentric, phase II study built with a two-stage Simon's design (NCT02505048). All patients provided written informed consent. Eligible patients were 18 years or older women with progressive HER2-breast cancer previously treated with at least one line of chemotherapy in the metastatic setting. Patients had a genomic $\mathrm{LOH}$-high score obtained from an available genome-wide human SNP array or a BRCA1/2 somatic mutation, without known $B R C A 1 / 2$ deleterious germline mutation. Patients harboring $S B R C A 1 / 2$ mutations could have come from genomics driven trials such as SAFIR02-Breast (NCT02299999), MOSCATO ${ }^{36}$ or PERMED (NCT02342158) trials. Other inclusion criteria comprised measurable disease (according to response evaluation criteria in solid tumors version 1.1 [RECIST v1.1]), an ECOG performance status 0 or 1, and a 21-day washout period from last chemotherapy or targeted therapy with resolution of all toxicities to grade $\leq 1$. Main exclusion criteria included known $g B R C A 1 / 2$ deleterious mutation, contraindication to rucaparib treatment, previous treatment with PARP inhibitor, less from 14 days from radiotherapy, spinal cord compression and/or symptomatic or progressive brain metastases, problem with intestinal absorption, severe or uncontrolled systemic disease, history of myelodysplastic syndrome, and hematopoietic function or organ impairment. The national ethics committee approved the study.

\section{Procedure and assessments}


Patients were treated with $600 \mathrm{mg}$ oral rucaparib twice a day until disease progression, unacceptable toxicity, or initiation of another antineoplastic treatment. Toxicity management and dose reduction followed summary of products characteristics recommendations and local standard practice. Clinical and laboratory examination were performed every 4 weeks after treatment initiation. The safety was assessed and graded by National Cancer Institute-common terminology criteria for adverse events version 4.03 ( $\mathrm{NCl}-\mathrm{CTCAE}$ v4.03) every 4 weeks from treatment initiation until the end of treatment. Assessment of response to treatment for therapeutic decision was based on investigator-reported measurements on target and non-target lesions and was done according RECIST v1.1 with computed tomography scans (CT-scans) or magnetic resonance imaging repeated every 8 weeks. For patients enrolled during the first stage of the study and patient with a response to treatment enrolled in the second stage, a central review was set-up to confirm investigator-reported measurements.

\section{Genomic LOH assessment}

The RUBY trial was tightly connected to the SAFIR trials. At RUBY initiation, all alive, non-germline BRCA1/2-mutated patients with a CytoScan HD or OncoScan CNV profile generated from metastatic tumor sample in SAFIR02Breast (NCT02299999) or SAFIRTOR (NCT02444390) studies, were screened for HRD. The SAFIR02-Breast/SAFIRTOR patient informed consent forms covered this screening phase. A local pathologist assessed biopsies from metastatic lesions to retain samples with more than $30 \%$ of cancer cells. DNA was extracted from 6 tissue sections (6- $\mu \mathrm{m}$ thick), using Nucleospin 8 8 Tissue kit (Macherey-Nagel, GmbH \& Co. KG, Germany) and a 7th tissue section was stained with hematoxylineosin. The AllPrep DNA/RNA Mini kit (Qiagen, Hilden, Germany) was used for isolation of DNA from frozen core biopsies, according to the manufacturer's protocol. The Qubit 2.0 Fluorometer (Quant-iT ${ }^{T M}$ dsDNA BR Assay Kit; Thermo Fisher Scientific) was used for DNA quantification according to the manufacturer's instructions. 300 to $500 \mathrm{ng}$ of DNA were used for DNA microarrays when more than 400 $\mathrm{ng}$ of extracted DNA were obtained. If DNA extraction procedure lead to less than $400 \mathrm{ng}$ of DNA, 10 to < $300 \mathrm{ng}$ of DNA were used for DNA microarray analysis. DNA microarrays analyses were performed using Affymetrix SNP6.0 technology (Thermo Fisher Scientific company): OncoScan ${ }^{\circledR}$ FFPE Assay Kit was used for FFPE tissue samples (designed for degraded DNA) and the CytoscanTM HD Array Kit was used for the fresh-frozen tissues. SNP probes were used both in the Oncoscan array and the Cytoscan arrays to provide DNA copy number variations.

Before being sent to Clovis Oncology, Affymetrix raw file (.cel) from SAFIR02/SAFIRTOR underwent a second pseudonymization procedure consisting of changing the SAFIR02/SAFIRTOR patient ID code with a new patient ID. A hexadecimal editor software (Frhed v1.6.0, http://frhed.sourceforge.net) was used to search and replace the patient ID code. To exclude any injury in the structure of the Affymetrix $®$ file after this procedure, each recoded file was tested in order to validate its integrity.

Finally pseudonymized array data files were sent to Clovis Oncology for HRD assessment. Percentage genome-wide LOH was calculated using the method previously described ${ }^{25}$. For each sample from the TCGA, SAFIR and SAFIR-TOR studies, LOH regions were inferred across the 22 autosomal chromosomes 
of the genome using the computed minor allele frequencies of the SNPs sequenced in the Affymetrix assays. LOH inference was based on Biodiscovery's implementation of the published ASCAT (allelespecific copy number analysis of tumors) methodology 37,38 . LOH regions spanning across $\geq 90 \%$ of a whole chromosome or chromosome arm were excluded from the calculation because these $\mathrm{LOH}$ events are likely due to non-HRD mechanisms ${ }^{39}$.

Hence for each tumor, the percentage of the genome with LOH was computed as 100 times the total length of non-excluded $\mathrm{LOH}$ regions divided by the total length of the interrogable genome.

In equation form:

$\%$ genome with $\mathrm{LOH}=100^{\star} \sum$ (lengths of non-excluded LOH regions) / (total length of genome with SNP coverage $-\sum$ (lengths of excluded LOH regions))

We prespecified a cutoff of $18 \%$ or more to define high genomic "LOH-high" score for breast carcinoma. This score was estimated to capture the top $25 \%$ of $\mathrm{LOH}$ scores based on previous analysis of The Cancer Genome Atlas (TCGA) ${ }^{2}$ microarray SAFIR01 and SAFIR02 microarray dataset $(n=675)$. The results shown here are in part based upon data generated by the TCGA Research Network: https://www.cancer.gov/tcga.

\section{Prognostic value of genomic LOH score}

Proportion of high LOH score was compared between early and mBC using TCGA dataset $(n=1624)$ and data from SAFIR02 trial $(n=620)$. Genomic LOH status was compared between TCGA and SAFIR-02 trial using chi-square or fisher exact test. Prognostic value of high genomic LOH was assessed only on patients from SAFIR02-Breast trial for which outcome data were available. OS was defined in SAFIR02Breast as the time from inclusion to death from any cause, and was estimated using the Kaplan-Meier method with $95 \% \mathrm{Cl}$. Patients alive at the time of analysis were censored at their last follow-up date. Univariable and multivariable analysis were performed using the log-rank test and Cox proportional hazards model, respectively. Significant factors (i.e $p \leq 0.05$ ) in the univariable analysis were included in the multivariable analysis.

\section{BRCA1 and BRCA2 deficiency prediction with HRDetect}

As an exploratory study, a whole-genome sequencing was performed in a sub-population of RUBY patients. Whole genome sequencing was performed as previously described ${ }^{11,40}$.

Data pre-processing. Whole-genome sequence reads were mapped to the Human genome build hg19 by using Burrows-Wheeler Aligner MEM algorithm (BWA v0.7.17) ${ }^{41}$ with the following parameter : -A 2. For compatibility with Genome Analysis Toolkit (GATK) ${ }^{42}$, the read group ID was attached to every read in the resulting alignment file (bam file) with the -R parameter, and shorter split hits were marked as secondary with -M. GATK FixMatelnformation was used to check mate-pair information between mates and fix if 
needed on a name sorted bam file. The duplicate reads were tagged by GATK MarkDuplicates (GATK4.1.4.0) using a position sorted bam file. Base quality score recalibration (BQSR) was performed with GATK BaseRecalibrator followed by GATK ApplyBQSR. In the first pass of BQSR method, the parameter -known-sites was used to specify three databases of known polymorphic sites:

1000G_phase1.indels.hg19.sites.vcf, Mills_and_1000G_gold_standard.indels.hg19.sites.vcf (downloaded from GATK Resource Bundle) and dbSNP144 ${ }^{40}$. The mean sequencing depth was assessed with deepTools plotCoverage (v3.3.1) ${ }^{43}$ on 50 million bases chosen randomly for each sample with the following parameters : -numberOfSamples 50000000 -ignoreDuplicates.

Somatic variant calling. The workflow described in GATK Best Practices for somatic short variant discovery was followed. In brief, somatic short variants (SNV and indel) calling was performed using GATK Mutect2, in tumor sample with matched normal. The -f1r2-tar-gz argument was used to output F1R2 counts that can be used to learn the orientation bias model. Also a germline resource file: af-onlygnomad.raw.sites.hg19.vcf (downloaded from GATK Resource Bundle) containing population allele frequencies of variants was used during the variant calling to filter common germline variants. The contamination in tumor sample can be estimated by a two steps method available in GATK. First, GetPileupSummaries was used with the file small_exac_common_3_b37.vcf (downloaded from GATK Resource Bundle) that has been lifted to hg19 with GATK LiftoverVcf. These genomic positions were used for two parameters of GetPileupSummaries: -intervals and -variant. The second step was performed by CalculateContamination. This tool calculates the contamination in the tumor from the results of GetPileupSummaries. In our case, we also used the matched normal results to get more precise estimations. Finally, GATK FilterMutectCalls was applied to filter variants due to contamination and/or due to strand/read orientation bias.

Germline variant calling. The workflow described in GATK Best Practices for germline short variant discovery was followed. In brief, germline short variants were called only in target genes by GATK HaplotypeCaller tool, in GVCF mode per sample. These GVCF files were merged using GATK GenomicsDBImport. Then GATK GenotypeGVCF was used for joint genotyping of tumor and normal samples. Only variants with one alternate allele were considered (--max-alternate-alleles 1). We used the Variant Quality Score Recalibration (VQSR) method of GATK to filter the germline insertions/deletions with --max-gaussians 4 and SNPs with -max-gaussians 6.

Variants annotation. ANNOVAR program (v20191107) ${ }^{44}$ was used to annotate variants. In this tool, the relevant transcripts are reported based on refGene database ${ }^{45}$. Population allele frequencies available in the 1000 Genomes Project ${ }^{46}$, the Kaviar ${ }^{47}$, the gnomAD ${ }^{48}$ and the Complete Genomics 69 (cg69) ${ }^{49}$ databases were taken into account. The functional consequences of coding, non-coding and splicing SNVs are predicted with FATHMM ${ }^{50}$ and dbscSNV ${ }^{51}$ algorithms. Also the clinical significance from ClinVar database ${ }^{52}$, the presence of the variant in COSMIC (https://cancer.sanger.ac.uk) ${ }^{53}$ and dbSNP150 40 are added. 
Somatic copy number variations. The bioconda package cnv_facets (v0.15.0, https://github.com/dariober/cnv_facets/) was used to detect allele-specific copy number variants (CNVs) in tumor sample compared to a matched normal sample. The core of this tool uses the Facets $\mathrm{R}$ package ${ }^{54}$ and predicts the tumor purity, ploidy and clonal heterogeneity. The list of parameters used is: -snp-mapq 15 -snp-baq 20 -snp-count-orphans --depth 254000 -cval 25400 -nbhd-snp auto -gbuild hg19 -rnd-seed 1234.

Structural rearrangements. We used a tool called Manta (v1.6.0 $)^{55}$ to detect somatic structural variants with defaults parameters. This algorithm is based on supporting paired and split-read evidence. Inversions are reported as breakends by default. The script converlnversion.py was applied to resulting vcf files to reformat inversions into single inverted sequence junctions.

HRDetect analysis. The HRDetect-pipeline was downloaded from the Github repository: https://github.com/eyzhao/hrdetect-pipeline and modified to be used for our study. The modified code is available at: https://github.com/gustaveroussy/hrdetect-pipeline. The somatic short variants, copy number variations and structural rearrangements were used as input of the HRDetect pipeline. Then the HRDetect scores were determined as described previously ${ }^{56}$.

\section{Outcomes}

To assess the efficacy of the rucaparib treatment, the co-primary efficacy endpoints were the clinical benefit rate (CBR) and if significant, the overall response rate (ORR) according to a hierarchical procedure. The CBR was defined as the proportion of patients whose best overall response was either a complete response, a partial response, or a stable disease lasting for at least 16 weeks. The ORR was defined as the proportion of patients whose best overall response was either complete or partial response. The secondary endpoints were PFS, OS, and safety. PFS was defined as the time from first dose of rucaparib to disease progression or death from any cause. OS was defined as the delay between the date of inclusion to death from any cause. The safety profile of rucaparib was characterized by the occurrence of adverse events during the on-treatment period (defined as the period from the time of first dose of study medications up to 30 days after the last dose).

\section{Statistical analysis}

Under the assumption of a minimum CBR of $20 \%$, we calculated that we needed to include 37 patients to achieve a power of $90 \%$ at a two-sided a level of $10 \% .41$ patients were required considering attrition of $10 \%$ of non-evaluable patients. A success rate $>20 \%$ was considered to have clinical relevance, and a success rate of $>40 \%$ was considered to have a high clinical interest.

We applied a two-stage Simon's design to stop the trial early for futility in case of low proportion of patients with clinical response. The first stage required 19 patients. We planned to close the study if less than 4 patients achieved a clinical benefit to rucaparib treatment as confirmed by the central review of CT- 
scans. Otherwise, we planned to continue the study until 41 patients were included. We required at least 11 of 37 evaluable patients to achieve a clinical response in order to claim for the success of the study.

We analyzed the main criteria in the population defined by patients who received at least one dose of treatment and with a first, post-baseline, tumor assessment according to RECIST v1.1 (per protocol population). Patient who discontinued rucaparib for toxicity before the first tumor assessment were kept in the efficacy population (modified intent-to-treat [mITT] population) and considered as failures.

CBR and ORR were reported by percentage with $95 \% \mathrm{Cl}$ calculated using the binomial Clopper procedure. The secondary endpoints PFS and OS were estimated using Kaplan-Meier methods on the mITT population. At the time of analysis of PFS or OS, data were censored at the date of last follow-up visit for patients without clinical disease progression assessment and alive at the time of analysis for PFS, and alive at the time of analysis for OS. Patients who received any dose of rucaparib were included in the safety analyses. The frequency and percentage of events were summarized according to the primary system organ classes (SOC) and by preferred term of the medical dictionary for regulatory activities (MedDRA, version 19.1). Specific attention was provided to the events with a grade $>2$.

Data analysis were performed using R (version 3.6.0) software.

\section{Declarations}

\section{Acknowledgements:}

We thank the patients and their families as well as all of the investigators and their staff involved in RUBY and SAFIR02/SAFIRTOR.

Clovis provided drug supplies and funding.

We thank Lilian Amrein who provided medical writing services on behalf of Unicancer.

\section{Author Contributions:}

A.P and F.A. designed the study, contributed to the writing of the paper and were involved in recruitment, clinical care and data collection.

O.T., M.A., MP.S., A.G., C.V. were involved in recruitment, clinical care and data collection.

P.J. contributed to the microarray data formatting

A.L. calculated the LOH score for all data sets and determined the LOH cutoff score

T.F. supervised biostatistical analysis regarding prognostic value of LOH score

V.S. supervised all statistical analyses related to outcomes. 
D.N. performed the central review of all study CT-scans.

K.D. was in charge of the whole genome bioinformatics analyses.

I.B. and C.C revised the value of the BRCA mutations for patients inclusions

N.D. processed samples and generated the whole genome sequences

M.J. is the project manager of the RUBY and SAFIR02 trial and centralized collected samples and data.

All authors approved the final manuscript and contributed to critical revisions of its intellectual content.

\section{Competing Interests statement:}

Fabrice André received research fundings and served as speaker / advisor (compensated to the hospital) for Roche, AstraZeneca, Daiichi Sankyo, Pfizer, Novartis, Lilly.

Anne PATSOURIS received consulting fees (e.g. advisory boards) and served as a speaker (both compensed to the hospital) for Pfizer, Lilly, received travel fees from Roche, Esai, Amgen, Pfizer.

Olivier TREDAN received research grant from Roche, MSD-Merck, BMS, personal fees from Roche, MSDMerck, Pfizer, Lilly, Astrazeneca, Sandoz, Novartis-Sandoz, Daiichi Sankyo and travel fees from Eli-Lilly, Pfizer, Novartis, Astra Zeneca.

Monica ARNEDOS received research grant from Eli-Lilly, honoria from Pfizer, Novartis, Astra Zeneca, Seattle Genetics and Abbvie, travel fees from Roche, Astra Zeneca and Novartis

Anthony GONCALVES received research fundings and served as speaker / advisor, compensated to the hospital for Astra Zeneca, Pfizer, Novartis, Roche, MSD, Lilly

Cecile VICIER received research grant from BMS and travel fees from Pfizer, Astellas and Roche.

Andrea LOEHR is affiliated to Clovis and a no competing interest to disclose otherwise.

Marie-Paule SABLIN NO DISCLOSURE

Khadija DIOP NO DISCLOSURE

Nathalie DROIN NO DISCLOSURE

Ivan BIECHE NO DISCLOSURE

Celine CALLENS NO DISCLOSURE

Daniel NENCIU NO DISCLOSURE

Pascal JEZEQUEL NO DISCLOSURE 
Thomas FILLERON NO DISCLOSURE

Valérie SEEGERS NO DISCLOSURE

Marta JIMENEZ NO DISCLOSURE

\section{References}

1. Deluche, E. et al. Contemporary outcomes of metastatic breast cancer among 22,000 women from the multicentre ESME cohort 2008-2016. Eur. J. Cancer Oxf. Engl. 1990 129, 60-70 (2020).

2. Cancer Genome Atlas Network. Comprehensive molecular portraits of human breast tumours. Nature 490, 61-70 (2012).

3. Farmer, H. et al. Targeting the DNA repair defect in BRCA mutant cells as a therapeutic strategy. Nature 434, 917-921 (2005).

4. Lord, C. J. \& Ashworth, A. PARP inhibitors: Synthetic lethality in the clinic. Science 355, 1152-1158 (2017).

5. Research, C. for D. E. and. FDA approves olaparib for germline BRCA-mutated metastatic breast cancer. FDA (2019).

6. Research, C. for D. E. and. FDA approves talazoparib for gBRCAm HER2-negative locally advanced or metastatic breast cancer. FDA (2019).

7. Robson, M. et al. Olaparib for Metastatic Breast Cancer in Patients with a Germline BRCA Mutation. N. Engl. J. Med. 377, 523-533 (2017).

8. Litton, J. K. et al. Talazoparib in Patients with Advanced Breast Cancer and a Germline BRCA Mutation. N. Engl. J. Med. 379, 753-763 (2018).

9. Diéras, V. C. et al. Phase III study of veliparib with carboplatin and paclitaxel in HER2-negative advanced/metastatic gBRCA-associated breast cancer. Ann. Oncol. 30, v857-v858 (2019).

10. Malone, K. E. et al. Prevalence and predictors of BRCA1 and BRCA2 mutations in a population-based study of breast cancer in white and black American women ages 35 to 64 years. Cancer Res. 66, 8297-8308 (2006).

11. Bertucci, F. et al. Genomic characterization of metastatic breast cancers. Nature 569, 560-564 (2019).

12. Turner, N., Tutt, A. \& Ashworth, A. Hallmarks of 'BRCAness' in sporadic cancers. Nat. Rev. Cancer 4, 814-819 (2004).

13. Birkbak, N. J. et al. Telomeric allelic imbalance indicates defective DNA repair and sensitivity to DNAdamaging agents. Cancer Discov. 2, 366-375 (2012).

14. Abkevich, V. et al. Patterns of genomic loss of heterozygosity predict homologous recombination repair defects in epithelial ovarian cancer. Br. J. Cancer 107, 1776-1782 (2012). 
15. Popova, T. et al. Ploidy and large-scale genomic instability consistently identify basal-like breast carcinomas with BRCA1/2 inactivation. Cancer Res. 72, 5454-5462 (2012).

16. Alexandrov, L. B., Nik-Zainal, S., Wedge, D. C., Campbell, P. J. \& Stratton, M. R. Deciphering signatures of mutational processes operative in human cancer. Cell Rep. 3, 246-259 (2013).

17. Davies, H. et al. HRDetect is a predictor of BRCA1 and BRCA2 deficiency based on mutational signatures. Nat. Med. 23, 517-525 (2017).

18. Gruber, J. J. et al. Talazoparib beyond BRCA: A phase II trial of talazoparib monotherapy in BRCA1 and BRCA2 wild-type patients with advanced HER2-negative breast cancer or other solid tumors with a mutation in homologous recombination (HR) pathway genes. J. Clin. Oncol. 37, 3006-3006 (2019).

19. Nadine M. Tung, M. E. R. et al. TBCRC 048: A phase Il study of olaparib monotherapy in metastatic breast cancer patients with germline or somatic mutations in DNA damage response (DDR) pathway genes (Olaparib Expanded).

20. Kristeleit, R. et al. A Phase I-II Study of the Oral Poly(ADP-ribose) Polymerase Inhibitor Rucaparib in Patients with Germline BRCA1/2-mutated Ovarian Carcinoma or Other Solid Tumors. Clin. Cancer Res. Off. J. Am. Assoc. Cancer Res. (2017) doi:10.1158/1078-0432.CCR-16-2796.

21. Abida, W. et al. Non-BRCA DNA Damage Repair Gene Alterations and Response to the PARP Inhibitor Rucaparib in Metastatic Castration-Resistant Prostate Cancer: Analysis From the Phase II TRITON2 Study. Clin. Cancer Res. Off. J. Am. Assoc. Cancer Res. (2020) doi:10.1158/1078-0432.CCR-20-0394.

22. Balasubramaniam, S. et al. FDA Approval Summary: Rucaparib for the Treatment of Patients with Deleterious BRCA Mutation-Associated Advanced Ovarian Cancer. Clin. Cancer Res. Off. J. Am. Assoc. Cancer Res. 23, 7165-7170 (2017).

23. Abida, W. et al. 846PD - Preliminary results from the TRITON2 study of rucaparib in patients (pts) with DNA damage repair (DDR)-deficient metastatic castration-resistant prostate cancer (mCRPC): Updated analyses. Ann. Oncol. 30, v327-v328 (2019).

24. Oza, A. M. et al. Antitumor activity and safety of the PARP inhibitor rucaparib in patients with highgrade ovarian carcinoma and a germline or somatic BRCA1 or BRCA2 mutation: Integrated analysis of data from Study 10 and ARIEL2. Gynecol. Oncol. 147, 267-275 (2017).

25. Swisher, E. M. et al. Rucaparib in relapsed, platinum-sensitive high-grade ovarian carcinoma (ARIEL2 Part 1): an international, multicentre, open-label, phase 2 trial. Lancet Oncol. 18, 75-87 (2017).

26. Frampton, G. M. et al. Development and validation of a clinical cancer genomic profiling test based on massively parallel DNA sequencing. Nat. Biotechnol. 31, 1023-1031 (2013).

27. Patsouris, A. et al. Abstract PD8-12: Mutational processes, genome evolution and outcome in metastatic breast cancers. Cancer Res. 78, PD8-12 (2018).

28. Razavi, P. et al. The Genomic Landscape of Endocrine-Resistant Advanced Breast Cancers. Cancer Cell 34, 427-438.e6 (2018).

29. Meeting Library | TBCRC 048: A phase II study of olaparib monotherapy in metastatic breast cancer patients with germline or somatic mutations in DNA damage response (DDR) pathway genes 
(Olaparib Expanded). https://meetinglibrary.asco.org/record/185171/abstract.

30. Coleman, R. L. et al. Rucaparib maintenance treatment for recurrent ovarian carcinoma after response to platinum therapy (ARIEL3): a randomised, double-blind, placebo-controlled, phase 3 trial. Lancet Lond. Engl. 390, 1949-1961 (2017).

31. Polak, P. et al. A mutational signature reveals alterations underlying deficient homologous recombination repair in breast cancer. Nat. Genet. 49, 1476-1486 (2017).

32. Nik-Zainal, S. et al. Mutational processes molding the genomes of 21 breast cancers. Cell 149, 979993 (2012).

33. Nik-Zainal, S. et al. Landscape of somatic mutations in 560 breast cancer whole-genome sequences. Nature 534, 47-54 (2016).

34. Morganella, S. et al. The topography of mutational processes in breast cancer genomes. Nat. Commun. 7, 11383 (2016).

35. Chopra, N. et al. Homologous recombination DNA repair deficiency and PARP inhibition activity in primary triple negative breast cancer. Nat. Commun. 11, 2662 (2020).

36. Massard, C. et al. High-Throughput Genomics and Clinical Outcome in Hard-to-Treat Advanced Cancers: Results of the MOSCATO 01 Trial. Cancer Discov. 7, 586-595 (2017).

37. Van Loo, P. et al. Allele-specific copy number analysis of tumors. Proc. Natl. Acad. Sci. U. S. A. 107, 16910-16915 (2010).

38. Addressing Aneuploidy and Nonaberrant Cell Admixture in Tumor Samples for Copy Number Variation (CNV) and Loss of Heterozygosity ( $\mathrm{LOH})$ Analysis.

http://resources.biodiscovery.com/white-papers/aneuploidy-and-nonaberrant-cell-admixture-intumor-samples.

39. Thiagalingam, S. et al. Mechanisms underlying losses of heterozygosity in human colorectal cancers. Proc. Natl. Acad. Sci. U. S. A. 98, 2698-2702 (2001).

40. Sherry, S. T. et al. dbSNP: the NCBI database of genetic variation. Nucleic Acids Res. 29, 308-311 (2001).

41. Li, H. \& Durbin, R. Fast and accurate short read alignment with Burrows-Wheeler transform. Bioinforma. Oxf. Engl. 25, 1754-1760 (2009).

42. DePristo, M. A. et al. A framework for variation discovery and genotyping using next-generation DNA sequencing data. Nat. Genet. 43, 491-498 (2011).

43. Ramirez, J.-M. et al. Prognostic relevance of viable circulating tumor cells detected by EPISPOT in metastatic breast cancer patients. Clin. Chem. 60, 214-221 (2014).

44. Wang, K., Li, M. \& Hakonarson, H. ANNOVAR: functional annotation of genetic variants from highthroughput sequencing data. Nucleic Acids Res. 38, e164 (2010).

45. O'Leary, N. A. et al. Reference sequence (RefSeq) database at NCBI: current status, taxonomic expansion, and functional annotation. Nucleic Acids Res. 44, D733-745 (2016). 
46. 1000 Genomes Project Consortium et al. A global reference for human genetic variation. Nature 526, 68-74 (2015).

47. Glusman, G., Caballero, J., Mauldin, D. E., Hood, L. \& Roach, J. C. Kaviar: an accessible system for testing SNV novelty. Bioinforma. Oxf. Engl. 27, 3216-3217 (2011).

48. Karczewski, K. J. et al. Variation across 141,456 human exomes and genomes reveals the spectrum of loss-of-function intolerance across human protein-coding genes. bioRxiv 531210 (2019) doi:10.1101/531210.

49. Drmanac, R. et al. Human genome sequencing using unchained base reads on self-assembling DNA nanoarrays. Science 327, 78-81 (2010).

50. Shihab, H. A. et al. Predicting the functional, molecular, and phenotypic consequences of amino acid substitutions using hidden Markov models. Hum. Mutat. 34, 57-65 (2013).

51. Jian, X., Boerwinkle, E. \& Liu, X. In silico prediction of splice-altering single nucleotide variants in the human genome. Nucleic Acids Res. 42, 13534-13544 (2014).

52. Landrum, M. J. et al. ClinVar: improving access to variant interpretations and supporting evidence. Nucleic Acids Res. 46, D1062-D1067 (2018).

53. Tate, J. G. et al. COSMIC: the Catalogue Of Somatic Mutations In Cancer. Nucleic Acids Res. 47, D941-D947 (2019).

54. Shen, R. \& Seshan, V. E. FACETS: allele-specific copy number and clonal heterogeneity analysis tool for high-throughput DNA sequencing. Nucleic Acids Res. 44, e131 (2016).

55. Chen, X. et al. Manta: rapid detection of structural variants and indels for germline and cancer sequencing applications. Bioinforma. Oxf. Engl. 32, 1220-1222 (2016).

56. Zhao, E. Y. et al. Homologous Recombination Deficiency and Platinum-Based Therapy Outcomes in Advanced Breast Cancer. Clin. Cancer Res. Off. J. Am. Assoc. Cancer Res. 23, 7521-7530 (2017).

\section{Tables}


Table 1: High genomic LOH score in metastatic (SAFIR02) and early (TCGA dataset) breast cancer.

Genomic LOH score

TCGA BRCA SAFIR 02

$(n=1624)$

$(n=620)$

Overall population

Low

$800(79.7 \%)$

$414(66.8 \%)$

High

$204(20.3 \%)$

206 (33.2\%)

$p<0.0001$

ER+/HER2- $(n=815)$

Low

$393(86.2 \%)$

$252(70.2 \%)$

High

$63(13.8 \%)$

$107(29.8 \%)$

$\mathrm{p}<0.0001$

$\operatorname{TNBC}(\mathrm{n}=356)$

Low

$47(41.6 \%)$

147 (60.5\%)

High

$66(58.4 \%)$

96 (39.5\%)

$p=0.0009$

ER: Estrogen receptor, LOH: Loss of heterozygosity, PR: Progesterone receptor, TNBC: Triple-negative breast cancer 
Table 2: Baseline demographic and disease characteristics

Characteristic

Patients, $\mathbf{N}$

(\%)

$(\mathrm{N}=40)$

Age, Median (range), years

$54(27-76)$

Phenotype (metastatic biopsy)

ER+ and/or PR+

$21 / 40$

52.5

TNBC

$19 / 40$

47.5

Genomic instability

High genomic $\mathrm{LOH}$

$35 / 40$

87.5

sBRCA1/2 mutation

$5 / 40$

12.5

Metastases

De novo

$6 / 39$

15.4

Number of metastatic site

$<3$

$15 / 39$

$\geq 3$

$24 / 39$

Visceral involvement

Liver and/or lung metastases

$33 / 39$

84.6

Liver metastases

$29 / 39$

74.4

ECOG Performance Status

0

$23 / 40$

57.5

1

$17 / 40$

42.5

Median of previous lines of chemotherapy

$2(1-6)$

Number of patients receiving platinum salts

$10 / 40$

ECOG: Eastern Cooperative Oncology Group, ER: Estrogen receptor, LOH: Loss of heterozygosity PR: Progesterone receptor, TNBC: Triple-negative breast cancer 


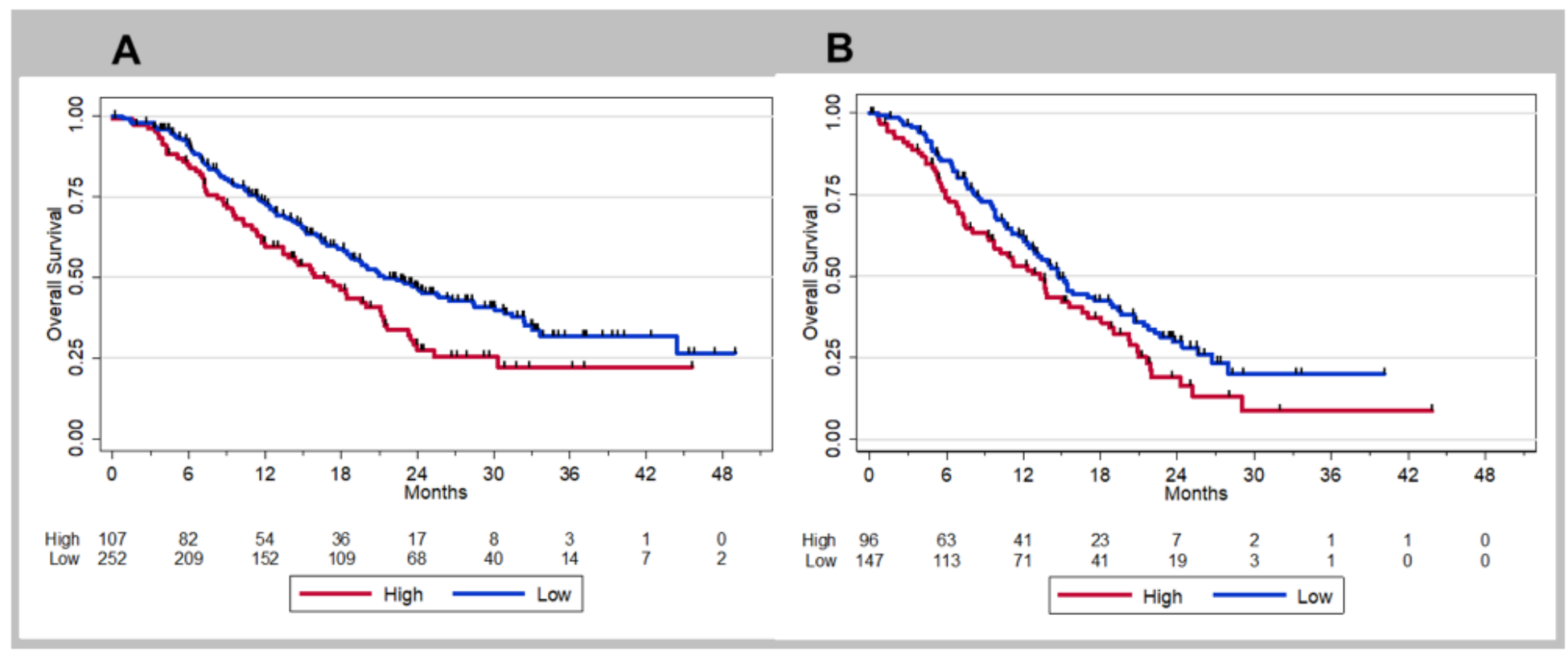

Figure 1

Overall survival based on LOH high versus low score in SAFIR02. A) ER+/HER2- breast cancer, adjusted $\mathrm{HR}=1.45$ (95\% Cl: 1.06-1.99), $\mathrm{p}=0.019$; Cox multivariate analysis adjusted on previous chemotherapy, liver metastasis (Yes versus No), number of metastatic site (<3 versus $\geq 3)$, and grade SBR ( $1 / 2$ versus 3$)$. B) Triple negative metastatic breast cancer. Adjusted HR = 1.323 (95\% Cl: 0.953-1.839), p = 0.095; Cox multivariate analysis adjusted on age at inclusion ( $\leq 50$ versus $>50$ ), liver metastasis (Yes versus No), and number of metastatic site (<3 vs $\geq 3$ ).
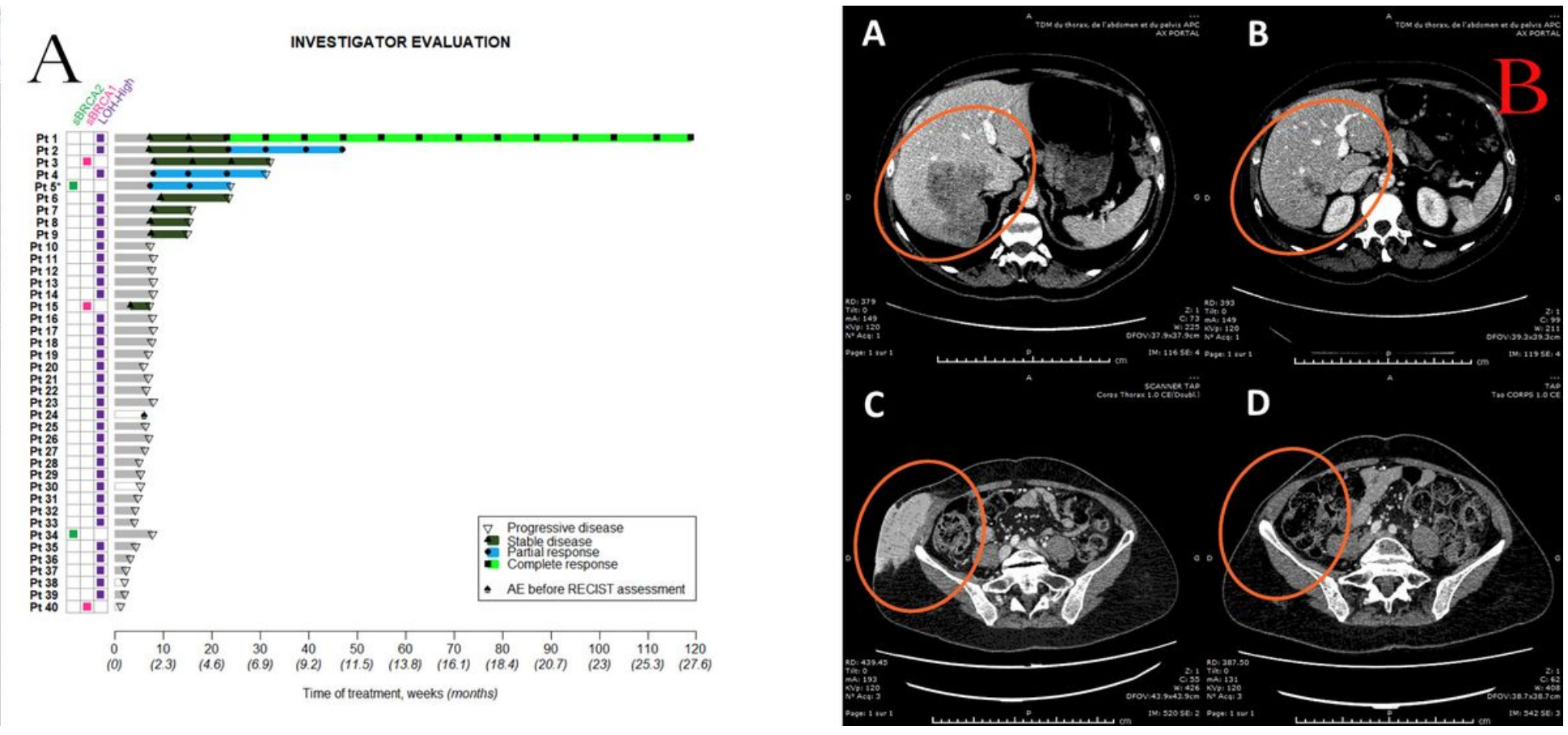

Figure 2 
Investigator response evaluation in RUBY. Figure 2a. Swimlane plot of duration of response in patients included based on high LOH score or somatic BRCA1/2 mutation with confirmed partial or complete RECIST responses. Each bar represents an individual patient with the length corresponding to the length of time on study drug. White bars represent patients that progressed or presented adverse event before the first RECIST assessment. *Patient 5 harbors a germline PALB2 mutation. LOH = loss of heterozygosity, sBRCA = somatic BRCA mutation, RECIST=Response Evaluation Criteria in Solid Tumors version 1.1, AE = adverse event. Figure 2b. CT-scan of 2 RUBY patients responding to rucaparib treatment. Portal venous phase of a right lobe liver metastasis for patient 4 at baseline evaluation $(A)$ and after 6 months of treatment (partial response) (B). Portal venous phase of muscle and skin metastasis located near the right iliac wing for patient 1 at baseline evaluation (C) and after 15 months of treatment (complete response) (D).

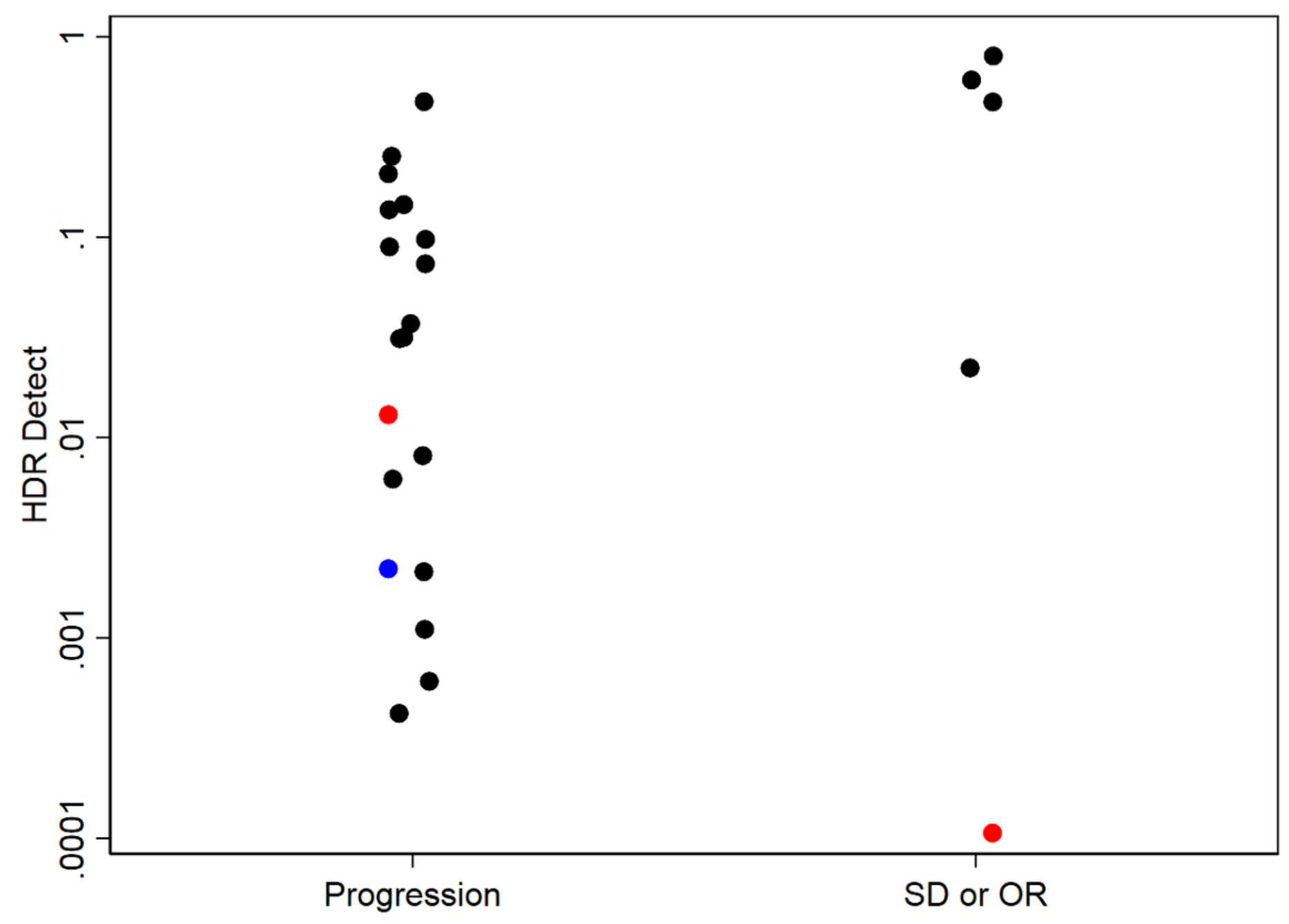

\section{Figure 3}

Correlation of HRDetect score and tumor response to rucaparib in RUBY On the 24 samples assessed for whole genome analysis, probability of BRCA1/2 deficiency exceeding 0.7 was significantly correlated to tumor response to rucaparib $(p=0.0362)$. Black points represent patients included on the basis of high 
LOH score, red points patients harboring somatic BRCA1 mutation and the blue point patient harboring a somatic BRCA2 mutation.

\section{Supplementary Files}

This is a list of supplementary files associated with this preprint. Click to download.

- RubySuplementarydatafilesSubmittedversionnaturecancer.docx 